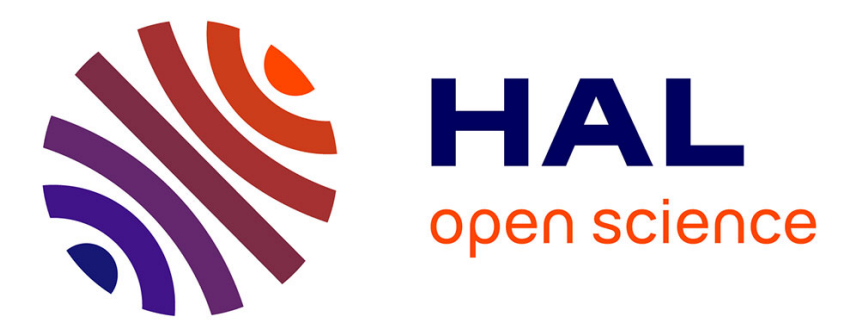

\title{
Second-harmonic microscopy of unstained living cardiac myocytes: measurements of sarcomere length with 20-nm accuracy.
}

Thierry Boulesteix, Emmanuel Beaurepaire, Martin-Pierre Sauviat, Marie-Claire Schanne-Klein

\section{To cite this version:}

Thierry Boulesteix, Emmanuel Beaurepaire, Martin-Pierre Sauviat, Marie-Claire Schanne-Klein. Second-harmonic microscopy of unstained living cardiac myocytes: measurements of sarcomere length with 20-nm accuracy.. Optics Letters, 2004, 29 (17), pp.2031-3. 10.1364/OL.29.002031 . hal00836429

\section{HAL Id: hal-00836429}

https://hal-polytechnique.archives-ouvertes.fr/hal-00836429

Submitted on 12 Oct 2013

HAL is a multi-disciplinary open access archive for the deposit and dissemination of scientific research documents, whether they are published or not. The documents may come from teaching and research institutions in France or abroad, or from public or private research centers.
L'archive ouverte pluridisciplinaire HAL, est destinée au dépôt et à la diffusion de documents scientifiques de niveau recherche, publiés ou non, émanant des établissements d'enseignement et de recherche français ou étrangers, des laboratoires publics ou privés. 


\title{
Second-harmonic microscopy of unstained living cardiac myocytes: measurements of sarcomere length with 20-nm accuracy
}

\author{
Thierry Boulesteix, Emmanuel Beaurepaire, Martin-Pierre Sauviat, and Marie-Claire Schanne-Klein \\ Laboratory for Optics and Biosciences, Centre National de la Recherche Scientifique-Institut National de la Santé et de la Recherche \\ Médicale, Ecole Polytechnique, 91128 Palaiseau, France
}

Received March 22, 2004

\begin{abstract}
We extend second-harmonic generation (SHG) microscopy to the measurement of sarcomere length in unstained living cardiac myocytes with 20-nm accuracy. We quantify individual sarcomere shortening in the presence of saxitoxin and find that it is in agreement with mechanical measurements of atrial tissue contracture. This functional application of SHG microscopy is generally applicable to quantify the physiological effects of drugs on contractile tissue. Our data also suggest that packed myosin heads in sarcomere thick filaments are responsible for the large second-harmonic endogenous signal in muscle tissue. (c) 2004 Optical Society of America

OCIS codes: 190.0190, 190.4160, 180.0180, 180.5810, 170.0170, 170.3880.
\end{abstract}

Second-harmonic generation (SHG) microscopy was demonstrated recently as a novel biological imaging technique that is well adapted to the specific observation of labeled membranes ${ }^{1,2}$ or unstained macromolecular structures composed of densely packed, well-organized proteins such as collagen or tubulin. ${ }^{3}$ A SHG endogenous signal in skeletal muscle was also reported and was attributed to the actomyosin complexes in sarcomeres, the muscular contractile units. $^{4-7}$

In this Letter we extend SHG imaging to live unstained cardiac myocytes, and we demonstrate that this technique allows accurate determination of subresolution variations of sarcomere length. In comparison, electron microscopy provides a resolution in the nanometer range but requires fixation and staining. Confocal fluorescence microscopy ${ }^{8}$ is less invasive and can be applied to living cells, but it requires the introduction of fluorophores, typically by exogenous staining or fusion protein (e.g., green fluorescent protein constructs) transfection. Moreover, the accuracy of length measurements with fluorescence images is very dependent on chromophore efficiency and photostability. In the following, we show that SHG microscopy allows direct observation of sarcomere contracture in unlabeled muscle cells and provides quantitative measurements with typical 20-nm accuracy. We show that this technique allows one to measure the physiological effects of a toxin on cardiac myocytes. Finally, images of sarcomere shortening provide evidence that the main source of intrinsic SHG signal lies in the myosin heads.

Sarcomeres are the elementary contractile units in cardiac myocytes and in skeletal muscle. ${ }^{9}$ They comprise thin actin filaments, with associated proteins, attached to the $\mathrm{Z}$ disks at both ends of the sarcomere (see Fig. 1). In the middle of the sarcomere, actin filaments overlap thick filaments of myosin, a protein with a long tail and globular heads. The myosin heads face in opposite directions on either side of a bare central region of the thick filaments, around the $\mathrm{M}$ line. During contraction, the myosin heads slide along the actin filaments, which results in sarcomere shortening, with no change in the length of the thin and thick filaments.

Atrial myocytes were obtained by enzymatic digestion of the sinoatrial region of an adult frog heart. ${ }^{10}$ In the resting state these myocytes are approximately $300-500 \mu \mathrm{m}$ in length and $3-5 \mu \mathrm{m}$ in diameter. They were kept at room temperature in a polystyrene tissue culture dish containing a Ringer solution, composed of (in millimoles per liter) $\mathrm{NaCl}$, $110.5 ; \mathrm{KCl}, 2.5 ; \mathrm{CaCl}_{2}, 2 ; \mathrm{MgCl}_{2}, 1$; Na pyruvate, 5; HEPES $(\mathrm{NaOH}), 10$; glucose 10 , albumine $(1 \mathrm{mg} / \mathrm{ml})$; and with $\mathrm{pH}$ 7.35. For imaging, myocytes were directly observed in the Ringer solution without staining.

SHG imaging was performed with a home-built laser scanning microscope comprising a femtosecond titanium sapphire laser (Mira, Coherent), galvanometer mirrors (GSI Lumonics), photoncounting photomultiplier modules (Electron Tubes), and laboratory-designed $100-\mathrm{MHz}$ counting electronics. Detection channels were implemented in both the reflected and the transmitted directions to allow for simultaneous detection of two-photon excited fluorescence and SHG signals by use of appropriate filters. ${ }^{2}$ For the experiments reported here we used a 0.9 -N.A., $60 \times$ water-immersion objective lens

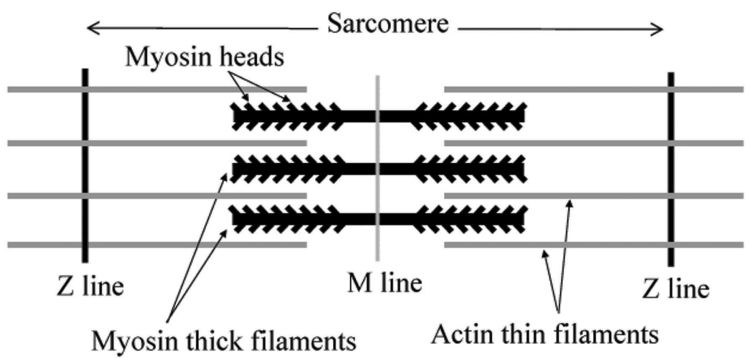

Fig. 1. Schematic representation of a sarcomere (see text). 
(Olympus) and achieved a spatial resolution of approximately $0.45 \mu \mathrm{m} \times 2 \mu \mathrm{m}$. Myocytes were illuminated with $20-40-\mathrm{mW}$ average power at $860 \mathrm{~nm}$, which resulted in no visible laser-induced morphological alterations of the cells. The SHG signal was detected in the forward direction through a 0.9-NA condenser (Olympus) by use of an interferential filter at the harmonic frequency (HQ430/20, Chroma) and a high-pass filter to block the laser excitation (E700SP, Chroma). We observed little two-photon excited fluorescence signal from the atrial myocytes in our experimental conditions, and no significant fading of the SHG signal over continuous illumination.

Typical SHG images are displayed in Fig. 2. Myocytes appear as striped structures that produce a readily detectable signal, typically peaking at $2 \times 10^{6}$ detected photons/s with $20-\mathrm{mW}$ excitation power. Similarly to what is found in SHG images of skeletal muscle ${ }^{5,7}$ bright areas are attributed to the myosin filaments and dark areas to the regions around the $\mathrm{Z}$ lines. Looking more precisely at individual sarcomeres, one can distinguish a darker region within the area that produces a SHG signal. This may partly be due to destructive interference, since opposite thick filaments on both sides of the M line exhibit a centrosymmetric orientation. ${ }^{5}$ However, the width of the SHG signal dip is significantly larger than the focal volume, which also indicates a local decrease of the density of harmonophores. Consequently, these images suggest that the bare central area in the thick filaments produces no SHG signal and that the SHG signal arises principally from the myosin heads.

In the following, we use the above-described method to quantify the physiological effect of the paralytic shellfish poisoning toxin saxitoxin (STX) on cardiac cells. STX blocks $\mathrm{Na}^{+}$channels in excitable cells. ${ }^{11}$ We studied this toxin using electrophysiological and mechanical techniques. We found that STX at 8-nM concentration inhibits $81.5 \pm 0.7 \%$ of $\mathrm{Na}^{+}$channels in frog auricle and induces a small contracture $(3-15 \%)$ at rest on isolated atrial fibers. In the SHG experiments STX $(8 \mathrm{nM})$ was added to the Ringer solution and images were recorded and compared with images of control myocytes (see Fig. 2). SHG images of myocytes in STX are similar to the control SHG images: STX does not affect cellular and sarcomeric integrity, and the SHG signal has the same order of magnitude. However, sarcomeres appear to be smaller in STX than in the control, as revealed by further analysis of the SHG images.

Figure 3 describes the procedure for analyzing SHG data. The signal profile along a single myocyte is extracted, and the series of double peaks is fitted with Gaussians of adjustable heights, center positions, and widths. This procedure allows one to determine four relevant lengths, as indicated in Fig. 3B and summarized in Table 1. The bare length is the distance between the two SHG peaks in a sarcomere. The dark length is the distance between two consecutive SHG peaks of neighboring sarcomeres, on both sides of the $\mathrm{Z}$ line. The full width at $1 / e^{2}$ of the SHG signal in a sarcomere is obtained as the sum of the bare length and the half-widths at
$1 / e^{2}$ of both adjacent SHG peaks. Finally, the total length of a sarcomere is obtained as the distance between two SHG peak pairs. All these lengths are determined as differences in the center positions of the Gaussian fits. The uncertainty is approximately $10 \mathrm{~nm}$ in peak localization and $15 \mathrm{~nm}$ in length determination. In a manner similar to what is commonly reported in single-molecule experiments, ${ }^{12}$ the achieved accuracy is well below the microscope resolution, owing to the remarkable contrast of the SHG signal. However, we observed small variations among sarcomeres within a single myocyte and from cell to cell. Finally, taking this sample variability

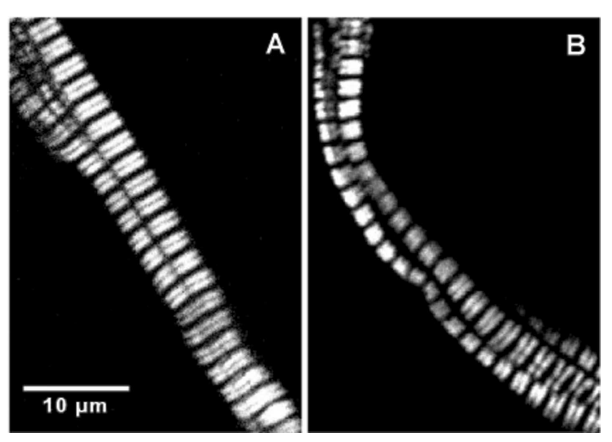

Fig. 2. SHG images from unstained atrial myocytes freshly isolated from an adult frog heart and kept in a Ringer solution. The sarcomeres are clearly delimited by thick black lines, and the SHG signal originates from the myosin filaments. A reduced SHG signal is obtained from the bare central region of the myosin filaments, which appears as a thin black line. A, myocytes in Ringer solution; $\mathrm{B}$, myocytes in Ringer solution containing STX $(8 \mathrm{nM})$. Excitation, $860 \mathrm{~nm}$, $20 \mathrm{~mW}$; 0.9-N.A. water objective. Image acquisition time, $2.3 \mathrm{~s}(390 \times 295$ pixels $/ 50 \mathrm{kHz})$.

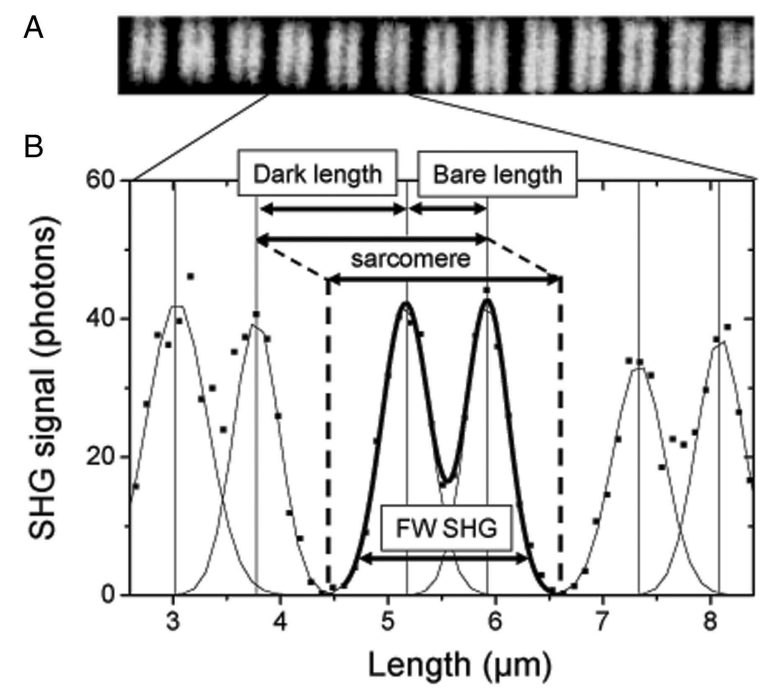

Fig. 3. SHG signal profile along a single atrial myocyte isolated from an adult frog heart. A, SHG image of a few sarcomeres selected in Figs. 2A and 2B; B, SHG signal (squares) extracted along a straight line in A. The solid curves correspond to fitting with multiple Gaussians. Individual Gaussians are also displayed to visualize the center of the peak (see solid vertical lines). The double arrows indicate the lengths reported in Table 1. 
Table 1. Sarcomere Characteristic Dimensions in the Absence and in the Presence of STX

\begin{tabular}{lccccc}
\hline & Samples $^{a}$ & Bare Length $^{b}$ & Dark Length $^{b}$ & Full Width SHG $^{b}$ & Sarcomere Length $^{b}$ \\
\hline Control & $6 / 60$ & $0.80 \pm 0.02$ & $1.51 \pm 0.02$ & $1.79 \pm 0.02$ & $2.31 \pm 0.02$ \\
STX $^{c}$ & $10 / 52$ & $0.75 \pm 0.02$ & $1.39 \pm 0.02$ & $1.82 \pm 0.02$ & $2.14 \pm 0.02$ \\
\hline
\end{tabular}

${ }^{a}$ Number of myocyte images analyzed and total number of sarcomeres fitted.

${ }^{b}$ All dimensions (in micrometers) are determined by Gaussian fitting of SHG images (see text and Fig. 3).

${ }^{c} \mathrm{STX}(8 \mathrm{nM})$ in Ringer solution.

into account, we obtained an overall measurement precision of approximately $20 \mathrm{~nm}$.

Table 1 reports the sarcomere data obtained in the absence and in the presence of STX $(8 \mathrm{nM})$ in the Ringer solution. We measured a resting sarcomere length of $2.31 \pm 0.02 \mu \mathrm{m}$ in the absence of STX, which is consistent with data already reported for frog auricle. $^{13}$ We measured a resting sarcomere length of $2.14 \pm 0.02 \mu \mathrm{m}$ in the presence of STX, which corresponds to a shortening of 7\%. This result is consistent with the global contracture measured in atrial fibers at rest by use of mechanical transducers. SHG microscopy is thus a direct and accurate technique for measuring contracture at the sarcomere scale.

Moreover, given the 20-nm measurement accuracy, we conclude that the SHG peak full width and bare length do not change significantly when STX is added, whereas the sarcomere length diminishes as a result of the shortening of the dark length. This is consistent with our assignment of the SHG signal to the myosin heads: the length of the thick myosin filaments does not change on contraction, nor does the size of the bare central area without myosin heads. Conversely, the overlap of the actin and myosin filaments increases, so the area with only thin filaments decreases. This area corresponds to the dark length measured in our experiments, which consistently decreases on contracture. Therefore these measurements provide evidence that the myosin filaments, and presumably the myosin heads, are responsible for the SHG signal, independent of their overlap with actin. This result is in agreement with reported measurements of genetically modified nematode muscle ${ }^{5}$ that demonstrated a significant contribution from myosin heavy chain B. This hypothesis on the origin of the SHG signal is also consistent with the large magnitude of the signal. As a coherent process, SHG scales as the square of the harmonophores' density and can be obtained only from noncentrosymmetric organizations. Consequently, endogenous SHG arises from densely packed, organized harmonophore distributions. Indeed, myofibril myosin filaments are packed in a quasi-crystalline arrangement and separated by approximately $50 \mathrm{~nm}$, and there are approximately 300 myosin heads per filament, ${ }^{9}$ which results in a high density of myosin heads in the focal volume.

In summary, we have extended SHG microscopy to the measurement of sarcomere length in unstained living cardiac myocytes with unprecedented $20-\mathrm{nm}$ accuracy. We demonstrated that this technique provides a quantitative measurement of the subcellular physiological response to a natural toxin, consistent with observed global tissue contracture. This technique is generally applicable to measurement of small sarcomere length variations on application of a toxin, a drug, or an ionic solution. Given the remarkable efficiency and contrast of SHG in sarcomeres, it is straightforward to extend this technique to measurements in intact tissue, including dynamic observation of contraction. In conclusion, SHG microscopy appears to be a promising tool in nanopharmocology of living unlabeled cardiac and muscular tissue.

The authors thank X. Solinas, M. Bierry, and J.-M. Sintes for technical assistance with the microscope design and assembly. M.-C. Schanne-Klein's e-mail address is marie-claire.schanne-klein@polytechnique.fr.

\section{References}

1. P. J. Campagnola, M.-de Wei, A. Lewis, and L. M. Loew, Biophys. J. 77, 3341 (1999).

2. L. Moreaux, O. Sandre, M. Blanchard-Desce, and J. Mertz, Opt. Lett. 25, 320 (2000).

3. W. R. Zipfel, R. M. Williams, R. Christie, A. Yu Nikitin, B. T. Hyman, and W. W. Webb, Proc. Natl. Acad. Sci. USA 100, 7075 (2003).

4. Y. Guo, P. P. Ho, H. Savage, D. Harris, P. Sacks, S. Schantz, F. Liu, N. Zhadin, and R. R. Alfano, Opt. Lett. 22, 1323 (1997).

5. P. J. Campagnola, A. C. Millard, M. Terasaki, P. E. Hoppe, C. J. Malone, and W. A. Mohler, Biophys. J. 82, 493 (2002).

6. S.-W. Chu, T.-M. Liu, C.-K. Sun, C.-Y. Lin, and H. J. Tsai, Opt. Express 11, 933 (2003), http:// www.opticsexpress.org.

7. M. Both, M. Vogel, R. H. A. Fink, and D. Uttenweiler, Proc. SPIE 5139, 112 (2003).

8. R. Littlefield and V. M. Fowler, Biophys. J. 82, 2548 (2002).

9. J. M. Squire, Curr. Opin. Struct. Biol. 7, 247 (1997).

10. M.-P. Sauviat, A. Colas, and N. Pages, BMC Pharmacol. 2, 15 (2002).

11. T. Narahashi, J. Pharmacol. Exp. Ther. 294, 1 (2000).

12. R. E. Thompson, D. R. Larson, and W. W. Webb, Biophys. J. 82, 2775 (2002).

13. P. W. Brandt, F. Colomo, N. Piroddi, C. Poggesi, and C. Tesi, Biophys. J. 74, 1994 (1998). 